JOURNAL OF COMPUTATIONAL PHYSICS 17, 349-357 (1975)

\title{
Waves in Periodically Structured Media
}

\author{
G. H. GoluB* \\ Computer Science Department, Stanford University, Stanford, California 94305 \\ L. JENNING* \\ Mathematics Department, University of Western Australia, Nedlands, Australia \\ AND \\ W. H. YANG \\ Department of Applied Mathematics and Engineering Science, \\ University of Michigan, Ann Arbor, Michigan 48104
}

Received March 26, 1974; revised September 17, 1974

\begin{abstract}
By the finite element method, the problem of steady-state wave motion in a 2-dimensional periodic medium is formulated in the form of a matrix eigenvalue problem. A matrix modification converts the large, sparse linear problem into a smaller, dense nonlinear matrix eigenvalue problem. The conversion makes the computation more efficient in data storage and in computing time. The dispersion curves of the lowest 10 modes are calculated. The propagation property of waves from the longest to modestly short wavelengths is discussed.
\end{abstract}

\section{INTRODUCTION}

Wave motion in a medium with periodic structure occurs in natural circumstances as well as in engineering applications. An example of the former is the dynamics of crystal lattices [1]. There are numerous engineering applications: Multiple reflection filters are used in modern optics to attain many useful effects [2]. Electromagnetic waves in dielectric layers provide features that lead to the designs of many interesting electronic devices [3]. Development of composite materials widens the imagination and practice of structure engineers [4]. More applications are yet to be developed. Hence, study of such wave motions may further enhance creative applications.

* The work of these authors was supported by the AEC and NSF. 
Mathematical modeling of the mentioned wave motions relates them to a common ground in terms of a class of linear partial differential equations with periodic coefficients. These coefficients are often discontinuous functions. By the Floquet theorem [5], the solution of such equations are quasiperiodic functions. The discontinuities in the coefficients cause the solution to possess limited differentiability. The classical Fourier analysis is either unsatisfactory or inefficient to handle these equations.

All these differential equations, when properly discretized, reduce to an algebraic eigenvalue problem of the form

$$
A \mathbf{x}=\lambda B \mathbf{x},
$$

where $A, B$ are real, symmetric, banded and positive definite matrices. However, the Floquet condition introduces complex elements in matrix $A$ outside its band. These complex elements appear as conjugate pairs at symmetric locations in $A$. They all contain the wave number as a parameter. This is an undesirable feature from computational point of view. The matrix $A$ now, although still sparse, is no longer a band matrix. It becomes Hermitian and it is a variable matrix with some of its elements contain the wave number.

In the case of 1-dimension periodic layers [6], the matrix has only a pair of off band complex elements. The solution is obtained by a rank one matrix modification method [7]. In the 2-dimensional case, the complex off band elements are still small in number $o(n)$ compared with the dimension $o(m)$ of the matrix $A(n \leqslant m)$. It is wasteful to treat $A$ as a full Hermitian matrix. It is not practical either since $m$ is usually large (e.g. $m \geqslant 200$ ) for an adequate approximation.

By using a rank $n$ matrix modification, the $m$-by- $m$ eigenvalue problem of Eq. (1) is reduced to a nonlinear eigenvalue problem of the form

$$
N(\lambda) \mathbf{x}=\mathbf{o}
$$

where $N(\lambda)$ is a $n \times n$ matrix with the eigenvalue parameter appears nonlinearly in its elements. Equation (2) is solved efficiently by an algorithm of Osborne [8].

The dispersion curves computed show similar behavior as that of 1-dimensional periodic layer in some respect in that they exhibit passing band and nonpassing band. But the situation is much more complicated because the mixing of various modes from multiple reflections and refractions of the waves through the complex structure of the medium. The modal transfer is indicated from the dispersion curves.

\section{FORMULATION}

Fiber reinforced composites consist of a homogeneous matrix (base) material in which a 2-dimensional array of filaments of a usually stiffer material is imbedded. 
For convenience of analysis, we shall assume the array be regularly arranged as shown in Fig. 1 and the wave propagation direction be perpendicular to the longitudinal direction of the filaments.

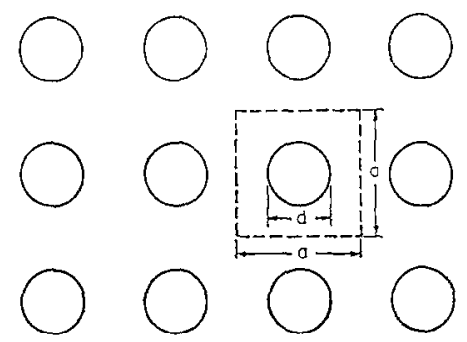

FIG. 1. Fiber reinforced composite.

The problem considered in this paper is to find a steady-state solution of the 2-dimensional displacement vector $\mathbf{u}(x, y, t)=(u \mathbf{i}+v \mathbf{j}) e^{i \omega t}$ that satisfies the differential equation

$$
\nabla(\alpha \nabla \cdot \mathbf{u})+\nabla \cdot[\beta(\nabla \mathbf{u}+\overline{\nabla \mathbf{u}})]=-\gamma \omega^{2} \mathbf{u},
$$

where $\nabla=(\partial / \partial x) \mathbf{i}+(\partial / \partial y) \mathbf{j}$ is a 2-dimensional operator and $\overline{\nabla \mathbf{u}}$ is the conjugate of $\nabla \mathbf{u} . \alpha, \beta$ are the Lame's elastic moduli and $\gamma$ is the density. They are constants for a material but take different values,

$$
(\alpha, \beta, \gamma)= \begin{cases}\left(\alpha_{b}, \beta_{b}, \gamma_{b}\right), & \text { in matrix material, } \\ \left(\alpha_{f}, \beta_{f}, \gamma_{f}\right), & \text { in filament, }\end{cases}
$$

for the composite. We may regard $\alpha, \beta, \gamma$ as discontinuous functions each of which varies as a step function between two values. Equation (3) is treated as a differential equation with variable coefficients which are discontinuous.

By requirement of continuity of the composite material, $u$ and $v$ must be continuous functions of $(x, y)$. The discontinuities in the coefficients cause $u, v$ to possess no differentiability at the interfaces of filaments and the base material. The classical Fourier representation of $u, v$ converges slowly. In practice, the truncated series oscillates near the interface, a phenomenon discussed by Gibbs [9].

The finite element representation [10] which uses localized basis functions can adequately approximate the class of limited differentiable functions at ease. In this paper, the piecewise linear bases over a triangular mesh of elements are used. The differential equation (3) is discretized through the use of the variational principle.

Since the material is 2-dimensional periodic, only one typical cell (Fig. 1) of the entire composite needs be considered. We shall let $x$ be the propagating direction 
of the waves. By symmetry, the domain of the mathematical problem is further reduced to the half-cell shown, with the triangular finite elements configuration, in Fig. 2.

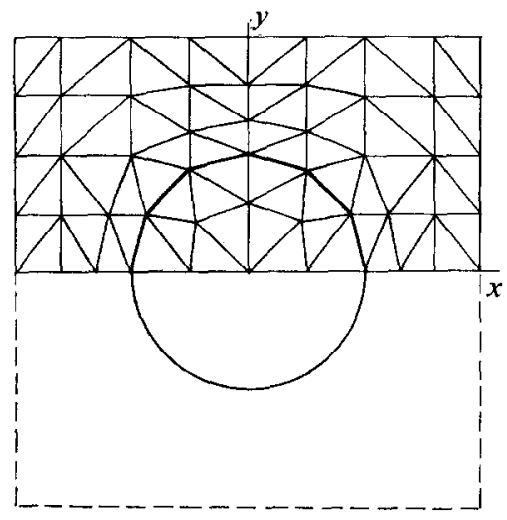

FIG. 2. Finite-element mesh configuration.

The boundary conditions on the half-cell domain are partially derived from the Floquet theorem [5]

$$
\begin{aligned}
\mathbf{u}(a / 2, y, t) & =e^{i q a} \mathbf{u}(-a / 2, y, t), \\
(\partial \mathbf{u} / \partial x)(a / 2, y, t) & =e^{i q a}(\partial \mathbf{u} / \partial x)(-a / 2, y, t),
\end{aligned}
$$

where $\mathrm{a}$ is the cell dimension and $q$ is the wave number. The other boundary conditions are from symmetry

$$
\begin{aligned}
v(x, 0) & =v(x, a / 2)=0, \\
(\partial u / \partial y)(x, 0) & =(\partial u / \partial y)(x, a / 2)=0,
\end{aligned}
$$

which physically implies vanishing normal displacement and shear stress along those boundaries.

The result of the finite element approximation is an algebraic eigenvalue problem [10]

$$
K \phi=\lambda M \phi
$$

where $K$ and $M$ are stiffness and mass matrices, respectively, $\lambda=\omega^{2}\left(\gamma_{b} / \beta_{b}\right)$ is the eigenvalue parameter and $\phi$ is the eigenvector which is the discrete representation of the displacement functions $u, v$. The matrix $M$ is banded. The Floquet conditions of (5), which relate the displacements of the nodes on the right edge to that on the 
left edge, introduce $2 n$ complex elements outside the band of $K$ otherwise having the same band width as $M$. In pictorial form,

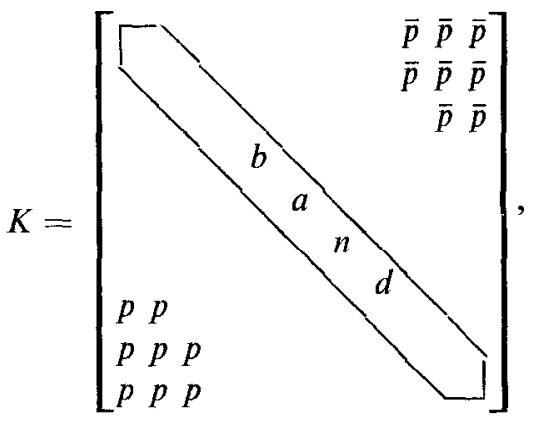

where $p$ 's and their complex conjugates $\bar{p}$ 's may appear at arbitrary locations in the matrix $K$ outside its band.

As it stands, $K$ is a Hermitian matrix of dimension $m \times m$ with 2 n complex elements. For the rather coarse element mesh size used in this paper, $m \approx 100$ and $n \approx 20$. For a more refined mesh, it is more likely that $n \ll m$. Hence the matrix $K$, although not banded, is very sparse. The algebraic eigensystem of (7) needs be solved repeatedly for various values of $q a$ ranging from 0 to $\pi$. The parameter $q$ appears only in the factors $\exp ( \pm$ iqa $)$ in the off band elements of $K$. It is, therefore, very wasteful and impractical to treat $K$ as a full Hermitian matrix. The special structure of $K$ as shown in (8) should be taken into consideration.

Each off band conjugate pair in $K$ can be generated by a rank-one matrix ww*, where $\mathbf{w}^{*}$ is the conjugate transpose of the vector

$$
\mathbf{w}=(0 \cdots 010 \cdots 0 p 0 \cdots 0)^{T}
$$

The locations of 1 and $p$ in the vector depend on the position of the element $p$ in $K$. The matrix $K$ can now be written in a form

$$
K=H+W W^{*},
$$

where $H$ is a real, symmetric, band matrix of order $m$ and $W$ is a $m \times n$ matrix of rank $n$. The algebraic eigenvalue problem now has the form

$$
\left(H+W W^{*}\right) \phi=\lambda M \phi
$$

which is a $m \times m$ sparse linear problem. 


\section{CONVERSION}

We shall convert the sparse $m \times m$ linear eigenvalue problem of (11) into an essentially full $n \times n$ nonlinear eigenvalue problem. The small dimension of the nonlinear problem is the prime motivation of the conversion.

Assume that $\lambda$ is not a zero of $\operatorname{det}(H-\lambda M)$ which is true for the problem considered. Equation (11) can be rewritten in the form

$$
\phi=-(H-\lambda M)^{-1} W W^{*} \phi .
$$

Although $\lambda$ is real, $\phi$ is in general complex, $\phi \in C^{m}$. Introducing a new variable $\psi \in C^{n}$ such that

$$
\psi=W^{*} \phi
$$

we have from (12).

$$
\psi=-W^{*}(H-\lambda M)^{-1} W \psi .
$$

Now, the original problem is converted into a nonlinear eigenvalue problem of the form

$$
N(\lambda) \boldsymbol{\psi}=\mathbf{0},
$$

where the $n \times n$ matrix,

$$
N(\lambda)=I+W^{*}(H-\lambda M)^{-1} W,
$$

is called the $\lambda$-matrix.

The eigenvalues and eigenvectors of (15) are obtained by the method of Osborne [8]. The method is iterative in nature. Once an eigenpair $\left(\lambda_{i}, \psi_{i}\right)$ is converged, $\lambda_{i}$ is the corresponding eigenvalue of the original problem (7). The corresponding eigenvector $\phi_{i}$ can be obtained from

$$
\phi_{i}=-\left(H-\lambda_{i} M\right)^{-1} W \psi_{i} .
$$

Note that $\left(H-\lambda_{i} M\right)$ is a symmetric band matrix. When this factor is implemented in the Osborne's method, the iterative procedure is quite efficient.

\section{The Method AND Algorithm}

The Osborne algorithm applied to this problem is briefly described as follows. For suitable choice of $\mathbf{r}$ and $\mathbf{s} \in C^{n}$, a scalar function $\rho(\lambda)$ is defined by

$$
\rho(\lambda) N^{-1}(\lambda) \mathbf{r}=\psi, \quad \mathbf{s}^{T} \boldsymbol{\psi}=1 .
$$


$\rho(\lambda)$ has zeros which are the eigenvalues of $N(\lambda)$. The zeros can be obtained by the Newton's iteration

$$
\lambda_{i+1}=\lambda_{i}-\rho\left(\lambda_{i}\right) / \rho^{\prime}\left(\lambda_{i}\right)
$$

It remains to find an expression for $\rho(\lambda) / \rho^{\prime}(\lambda)$.

Differentiating (18), we produce

It follows that

$$
\begin{gathered}
d \Psi / d \lambda=\rho(\lambda)(d / d \lambda) N^{-1}(\lambda) \mathbf{r}+\rho^{\prime}(\lambda) N^{-1}(\lambda) \mathbf{r}, \\
\mathbf{s}^{T}(d \Psi / d \lambda)=0 .
\end{gathered}
$$

$$
\rho(\lambda) / \rho^{\prime}(\lambda)=-\left[\mathbf{s}^{T} N^{-1} \mathbf{r} / \mathbf{s}^{T}\left(d N^{-1} / d \lambda\right) \mathbf{r}\right]
$$

Note that

$$
d N^{-1} / d \lambda=-N^{-1}(d N / d \lambda) N^{-1}
$$

For the special $N(\lambda)$ in $(16)$,

$$
d N / d \lambda=W^{*}(H-\lambda M)^{-1} M(H-\lambda M)^{-1} W .
$$

Hence, we have an explicit expression for $\rho(\lambda) / \rho^{\prime}(\lambda)$. Calculation of $(H-\lambda M)^{-1} W$ and $(H-\lambda M)^{-1} M$ is done by a band LU decomposition routine.

The iterative algorithm at the $i$ th step is

1. $\Psi^{(i+1)}=N^{-1}\left(\lambda_{i}\right) \mathbf{r}^{(i)}$

2. $\mathbf{t}^{(i+1)}=-N^{-1}\left(\lambda_{i}\right)(d N / d \lambda) \psi^{(i+1)}$

3. $\lambda_{i+1}=\lambda_{i}-\left(\mathbf{s}^{T} \Psi^{(i+1)} / \mathbf{s}^{T} \mathbf{t}^{(i+1)}\right)$

4. $\mathbf{r}^{(i+1)}=\mathbf{t}^{(i+1)} / \mathbf{s}^{T} \mathbf{t}^{(i+1)}$

A convenient choice of $\mathbf{s}$ is $\mathbf{e}_{k}$, the unit vector in coordinate $k$, where $k$, the index, corresponds to the largest absolute element of $t^{(i+1)}$. We need an initial pair $\lambda_{0}, \mathbf{r}^{(0)}$ to start the iteration. Physically, we expect that $\lambda(q)$ is continuous over the interval $[o, \pi]$ for each mode. When $\lambda\left(q_{j}\right)$ and $\psi\left(q_{j}\right)$ are known, we may use them as initial estimates for $\lambda\left(q_{j} \pm h\right)$, where $h$ is a small increment of $q$. From one mode to another, a search procedure is needed. It is easy to search $\lambda$ at $q=o$ and $\pi$. Thus we can proceed the calculation with either increasing or decreasing $q$ for each mode.

\section{RESUlts AND Discussion}

The model cell for the numerical results presented in this paper has cell dimension $a$ and filament diameter $d$. The ratio $d / a=0.5$ is chosen. The ratios of other parameters are $\alpha_{f} / \alpha_{b}=\beta_{f} / \beta_{b}=4.0, \gamma_{f} / \gamma_{b}=3.0$. The dispersion relations computed are depicted in Fig. 3. 


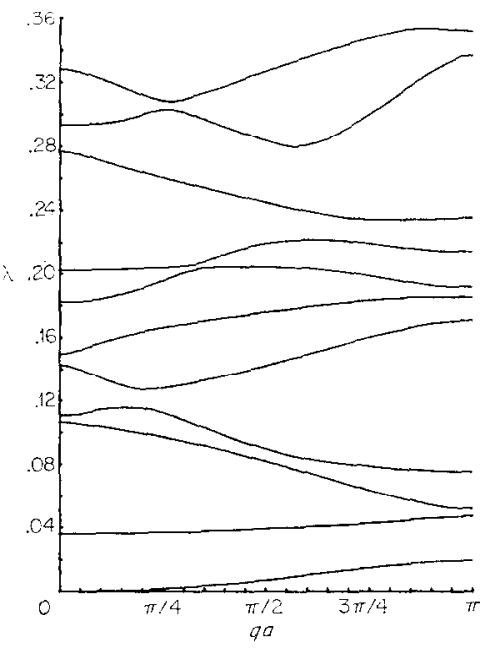

FIG. 3. Dispersion relations.

The results exhibit marked difference from the dispersion relations for the layered medium shown in [6]. The waves in [6] are 1-dimensional in that shear waves are not present. For the problem in this paper, both longitudinal and shear waves are propagated through the medium. Unlike a homogeneous material in which the longitudinal and shear waves propagate separately in different speeds, the waves in the composite are reflected and refracted at each interface of a filament and the matrix creating complicated mixing of different waves.

In the case of a layered medium, the waves have a property that the group velocity is zero at $q a=0, \pi$. The group velocity is the slope of the dispersion curve. This is experimentally verified as the ends of the pass bands and no-pass bands. The fiber reinforced composite has the similar behavior at low frequencies. At higher frequencies, the zero group velocity could occur anywhere between $q a=0$ and $q a=\pi$ as shown in Fig. 3 .

The dispersion curves shown do not intersect. It is demonstrated in Fig. 3 that two of such curves approach each other and then repell from each other. Physically, this indicates an interaction of two adjacent modes. Such interaction between modes does not exist in the 1-dimensional case.

\section{REFERENCES}

1. L. Brillouin, "Wave Propagation in Periodic Structures," 2nd ed., Dover, New York, 1953.

2. M. BORN AND E. Wolf, "Principles of Optics," Pergamon, 1959.

3. R. E. Collin, "Foundations for Microwave Engineering," McGraw-Hill, New York, 1966.

4. E. H. LeE, "Dynamics of Composite Material," ASME, 1972. 
5. E. L. INCE, "Ordinary Differential Equations."

6. W. H. Yang ANd E. H. LeE, J. Appl. Mech. 73-APMW-40.

7. G. H. Golub, SIAM Rev. 15 (1973).

8. M. R. Osborne, Comput J. 7 (1964).

9. R. W. Hamming, "Numerical Methods for Scientists and Engineers," McGraw-Hill, New York, 1962.

10. J. T. Oden, "Finite Elements of Nonlinear Continua,” McGraw-Hill, New York, 1962. 